\title{
Application of Gamma functions to the determination of
}

\section{unit hydrographs}

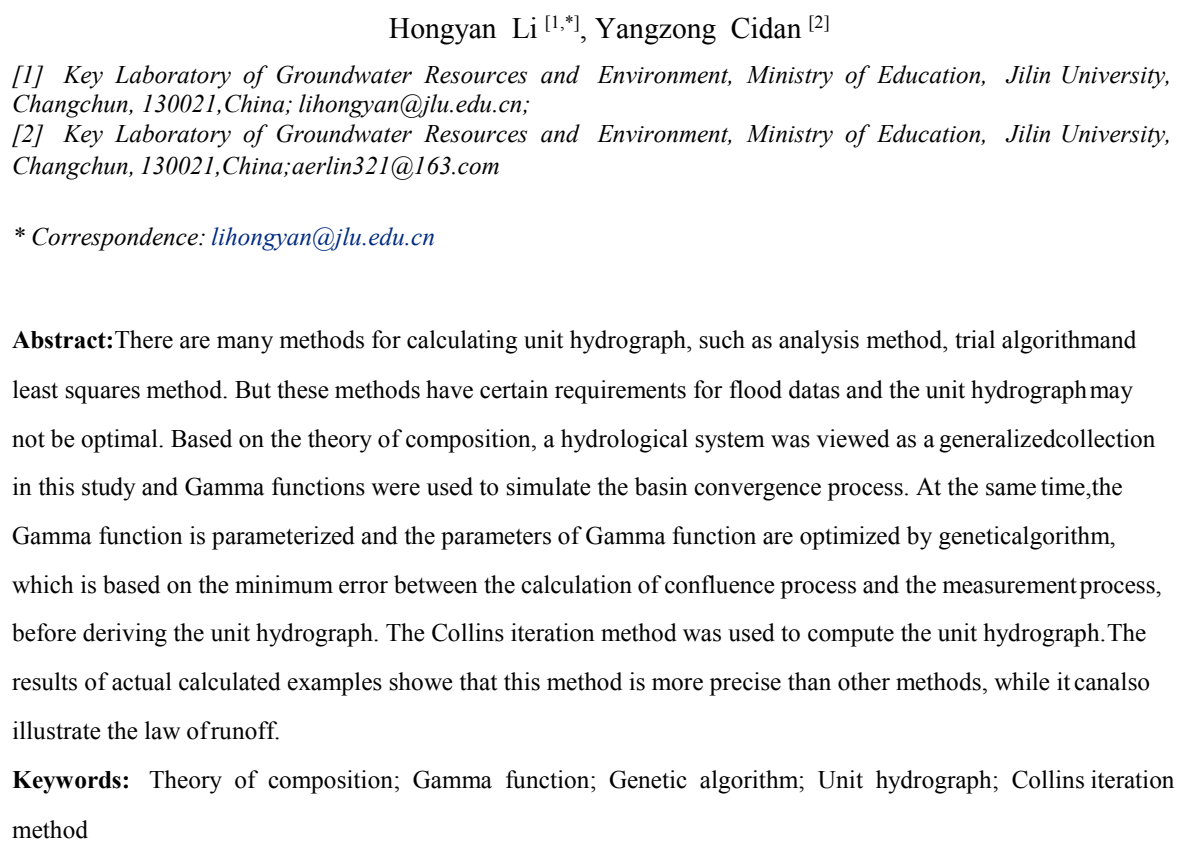

\section{Introduction}

The unit hydrograph is an important method for simulating the flow concentration of a conceptual hydrological model; it was proposed by Sherman (1932). In actual applications, the derivation of the unit hydrograph is still an important component when forecasting the basin rainfall and runoff.

A unit hydrograph (Viessman, 1989; Raghunath, 2006) refers to the unit net constant rainfall uniformly distributed over a watershed of unit surface and for unit duration. Periods of 1,3 , 6 , and $12 \mathrm{~h}$ can be selected and the unit rainfall (runoff depth) is generally $10 \mathrm{~mm}$. The actual net rainfall often does not equal 1 unit for these time periods, so it is necessary to make two basic assumptions when calculating the watershed flow concentrationprocess.

(a) Assumption of linear hydrological system: if the unit period rainfall is $\mathrm{k}$ units, the formation of the flow process is $\mathrm{k}$ times the unit hydrograph ordinate.

(b) Superposition assumption: if the rainfall lasts m periods, the formation of the flow process is the superposition of each rainfall period.

Based on the above assumptions, the basin outlet section discharge hydrograph can be expressed as: 


$$
\begin{aligned}
& Q_{i}=\sum_{j=1}^{m} h_{i} q \underset{i-j+1}{ }\left\{\begin{array}{l}
i=1,2, \quad, l \\
j=1,2, \quad, m
\end{array}\right. \\
& \mid \begin{array}{l}
i-j+1=1,2, \quad, n
\end{array}
\end{aligned}
$$

where $Q_{i}$ is the basin outlet section of each period discharge in $\mathrm{m} 3 / \mathrm{s}$;

$$
\begin{aligned}
& h_{j} \text { is the rainfall in each period in mm; } \\
& q_{i-j+1} \text { is the ordinate of the unit hydrograph in each period in } \mathrm{m} 3 / \mathrm{s} ; \\
& i \text { is the number of periods for the basin outlet section flow hydrograph; } \\
& j \text { is the net number of rainfall periods;and }
\end{aligned}
$$

In essence, the unit hydrograph is the characteristic of watershed concentration in the form of discharge hydrograph, i.e. concentration curve (d.johnstone, 1949). The calculation method of unit hydrograph confluence takes the basin as a whole and assumes that the net rain is uniformly distributed over the whole basin, without considering the inhomogeneity within the system; the basin confluence system is a linear time-invariant system, and at the same time, it is viewed that the net rainfall and the formation of the flow process are in agreement to superposition relationship. Therefore, the essential characteristics of the unit hydrograph are lumped resistance, linearity, and time invariance.

Conceptually, the unit hydrograph is a linear time-invariant basin system with a convergent flow curve. However, the physical mechanism of the watershed conflux is not considered by the method used to derive the unit hydrograph (Ramirez, 2000). The principle used to calculate the unit hydrograph is based on the system input (rainfall), which is converted using the unit hydrograph to determine the system response output (outlet section flow process), where the error is minimized. The traditional methods of derivation are as follows.

Analytical approach: the basin outlet section of the surface runoff is $Q_{1}, Q_{2}, \quad, Q_{l}$, the rainfall process is $h_{1}, h_{2}, \quad, h_{m}$, where Eqn. (1) comprises $q_{1}, q_{2}, \quad, q_{n}$ unknown linear algebraic equations. The solutions of the equations can be obtained using a unithydrograph.

$$
\begin{aligned}
& -h_{j q} \\
& q_{i}=\underset{h_{1}}{Q_{i}} \sum_{i-j+1}^{i=1,2,}, n
\end{aligned}
$$

10

where $\mathrm{n}$ is the number of periods of the unit hydrograph and $n=l-m+1$.

In theory, there are no errors with the analytical approach when using the rainfall runoff measurements. This approach can obtain the correct answer if the watershed conflux conforms to a 
linear time-invariant system (when the convergence of watershed system meets multiple proportions and superposition assumption, for a linear system). However, (1) the data measurements have errors and, (2) rainfall-runoff system is not a linear time-invariant system. Due to the accumulation of errors, unreasonable solutions often occur. For example, the unit hydrograph may be irregular or appear to be negative.

(b) Trial and error: the unit hydrograph is assumed to be $q_{i}^{\prime}$. The flow $Q^{\prime}$ determined by the unit hydrograph is then compared with the measured flow ${ }^{Q}$. The unit hydrograph $q_{i}^{\prime}$ is produced when the error between Q' and Q satisfies certain error.

The trial and error method proposed by Collins (Collins, 1939) uses an iterative strategy. For a period of uneven net rainfall, the computational convergence is fast if the time period is large. However, the Collins iterative method has the following disadvantages: (1) the iterations are based on the initial unit hydrograph, but there is no strict method for selecting the initial unit hydrograph; and (2) trial-error approach does not necessarily converge to a solution.

(c) Least squares method: the measured surface runoff is assumed to be $Q$ and the error is as follows: $\varepsilon=Q-Q^{\prime}$. If $\sum \varepsilon^{2}=\sum(Q-Q)^{2} \rightarrow$ min , we try to convert Eqn. (1) into a normal equations system where the number of equations is equal to the variables. The optimal estimation of $q_{i}$ can be solved using the least squares method. The theory of the leastsquares method is better, but the results are sometimes fluctuating ornegative.

There are many methods for determining unit hydrographs, e.g., the $\mathrm{Z}$ transform method and the harmonic analysis method (Dooge, 1973). A previous study (Hanson and Johnson 1964 ) classified and compared the usual unit hydrograph calculationmethods.

In recent decades, the use of probability distribution functions (pdfs) to develop synthetic unit hydrographs (SUH) has received much attention because of its similar properties to unit hydrographs. First, the type of unit hydrograph needs to be subjected to mathematical analysis. Typical functions, such as a parabola P-III (Yuan, et al., 1991) (Zhai and Li, 2004)curve, can be used to describe the unit line and a mathematical model of the unit hydrograph can be established. A previous study (Bhunya, et al., 2007) explored the potential of using four popular pdfs, i.e., two-parameter Gamma, three-parameter Beta, two-parameter Weibull, and one-parameter Chi-square distribution, for deriving a SUH. The Gamma functions are the most widely used functions (Singh, 2005, 2009; Bhunya, et al., 2003). This approach aims to determine the relationship between each variable in a unit hydrograph, which facilitates a more in-depth analysis of a unit hydrograph.

In the present study, we used Gamma functions to describe a unit hydrograph and determined why a unit hydrograph may follow this distribution. The Gamma function parameters were 
optimized using a genetic algorithm. Finally, the unit hydrograph was obtained using the Collins iterative method.

For a specific basin, the confluence time of a flood is relatively stable and can be determined according to the flood datas. Therefore, the use of gamma function to derive unit hydrograph is only in the trial calculation of parameters $\beta$ and $k$. The unit hydrograph expressed by gamma function based on the combination of these parameters is optimal, which is compared with the use of P-III function to adjust its statistical parameters. The principle of hydrological frequency calculation by line fitness of numbers (mean, variation coefficient $\mathrm{Cv}$ and skewness coefficient $\mathrm{Cs}$ ) is very similar. Meanwhile, the time interval $t$, parameters and values of gamma function can parameterize the regional characteristics of river basin confluence, which hasthe important significance for this study.

\section{Mathematical model and method}

\subsection{Mathematical model}

In spatial or temporal physical entropy-based modeling of hydrology and water resources, the cumulative distribution function (CDF) of a design variable (e.g., a flux or a discharge) is analyzed in terms of its concentration (e.g., stage of flow) (Cui, et al., 2012). The theory of composition proposed by Zhang (2003) provides a model and a uniform calculation method for studying the composition of things. This theory considers the analysis of three concepts, i.e., the general set, the distribution function, and the degree of complexity. This theory is also considered the most highly approved principle followed by random systems, i.e., the entropyprinciple.

The variable $x$ is continuous and random, and can be viewed as a general set of flag variables. If the pdf $f(x)$ of $x$ agrees with the following function:

$$
f(x)=\frac{\beta^{k}}{(k-1) !} x^{k-1} e^{-\beta x}, x>0
$$

Then the pdf follows a Gamma distribution, where $\beta$ and $k$ are shape and scaleparameters. This is one of the famous Pearson pdfs, which is known as a Pearson type III distribution. The curve has a peak with a left-right asymmetry. In nature, many phenomena follow this distribution. In China, hydrological studies often use the Pearson type III distribution to simulate hydrological data series, because it has a greater than or equal to zero lower bound on the variable requirements and its elasticity is greater than the normal distribution (Ye and Xia, 2002). This choice is based on experience, but it lacks a theoretical justification.

Using entropy theory, a previous study (Zhang, 2003) described the physical form of this distribution. By analyzing the structure of Eqn. (3), is not difficult to show that it has a negative exponential distribution, which is a part of the exponential function, and it also has the characteristics of a power function in a Pareto-family distribution. The exponential distribution 
corresponds to the constraints on the invariant algebraic average of the flag variables, while the power function corresponds to the constraints on the invariant geometric mean. It may be speculated that the constraints on the Gamma distribution are the fixed algebraic average and geometric means of the variables.

In this study, $f(x)$ is the pdf of a positively defined random variable, i.e.,

$$
\int_{1=0}^{\infty} f(x)
$$

$u$ represents the algebraic average of variables, thus

$$
u=\int_{0}^{\infty} x f(x) d x
$$

while $v$ is the geometric mean of the random variable $x$, which can be expressed as the algebraic average of the logarithm, i.e.,

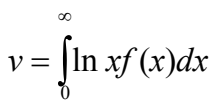

The entropy of the random variable ${ }^{x}$ can be written as

$$
H=-\int_{0}^{\infty} f(x) \ln x f(x) d x
$$

Given the constraints in Eqns. (4), (5), and (6), the Lagrange method can be used to estimate the distribution function $F$ based on the maximum entropy to determine the distribution function. Thus, $F$ is defined as follows:

$$
F=-\int_{0}^{\infty} f \ln f d x+C_{1}\left(\int_{0}^{\infty} f d x-1\right)+C_{2}\left(\int_{0}^{\infty} x f d x-u\right)+C_{3}\left(\int_{0}^{\infty} \ln x f d x-v\right)
$$

Where, $C_{1}, C_{2}$, and $C_{3}$ are undetermined constants. The entropy principle demands that the value of $F$ is maximal. The partial derivative of $f(\bullet)$, i.e., the partial derivative is 0 , can be obtained using Eqn (8). The results are as follows.

$$
f(x)=\exp \left(C_{1}-1+C_{2} x+C_{3} \ln x\right)
$$

This formula can be used to obtain the distribution function. It is the product of the power function and the exponential function, and its form is identical to a Gamma function.

Hydrological data are random variables that exceed zero. If the hydrological processes are stationary, then the algebraic average and geometric mean of the hydrological characteristics variable can be approximated as a fixed value. For example, a mean basin annual runoff is basically stable (the algebraic average is constant), so the probability of a major flood occurring is 
small, and most floods are close to the normal value of the accumulated years (the geometric mean is constant). However, the uncertainty of a different type of flood occurring each time is maximal. Thus, the complexity of the outcome is maximized. This is consistent with the following: "In a generalized (objective, system, sampling experiment), if the algebraic average and geometric mean of the variables (values of statistical indicators) are constant and the complexity is maximal, then we can conclude that the probability (the percentage) of the flag value (all the values of the variables) for each individual must obey a Gamma distribution (Pearson type III distribution) (Zhang, 2003).”

At the same time, a lot of practical experiences showed that Gamma function can reflect the characteristics of flood probability. In the view of this, the unit line

$q_{i}$ is defined as Gamma function.

$$
q=\frac{\beta^{k}{ }_{i}{ }^{k-1}}{(k-1) !} e^{-\beta i}, i=1,2, \quad, n
$$

When the parameters of $\beta$ and $k$ value were different types, the line type of $q_{i} \quad q_{i}$ was different, as shown in Fig. 1.

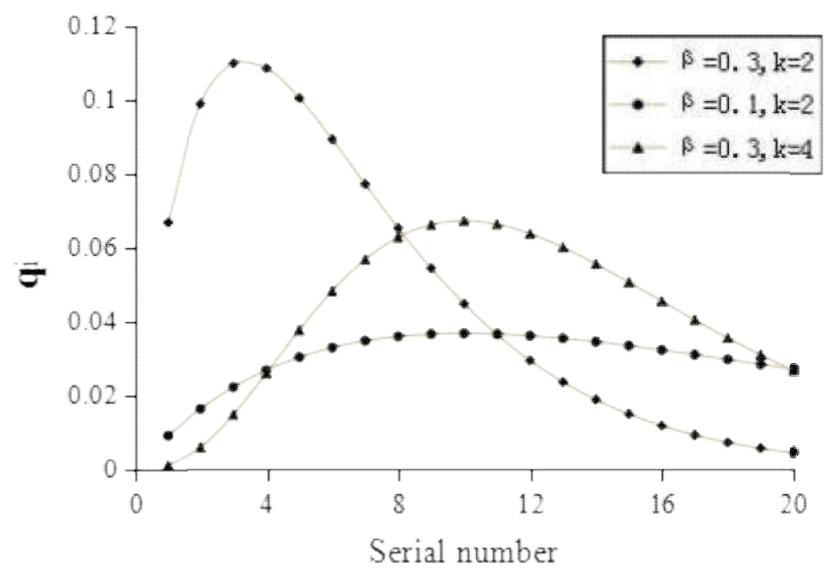

Fig. 1 The change of function under different parameters

\subsection{Method}

\subsubsection{Genetic algorithms}

Genetic Algorithms (GAs) is an effective global search method, which simulates natural selection and genetic mechanism. This method of searching the optimal solution of the problem through natural evolutionary process has been applied in many problems such as function 
optimization and combinatorial optimization. Genetic algorithm can automatically acquire and accumulate the knowledge of search space in the search process, and adaptively control the search process to find the best solution(Davis,1991;Michalewicz,1996). The genetic algorithm regards a family of randomly generated feasible solutions as the parent population, takes fitness function (objective function or one of its transformation forms) as the measurement of the ability of the parent individual to adapt to the environment, generates the offspring individual through selection and hybridization, and then mutates the latter, eliminating the fittest and the fittest, so that the individual adapts to the environment through repeated evolutionary iterations. With the continuous improvement of ability, excellent individuals keep approaching the optimum point(Yuan,2002). After several generations, the algorithm converges to the best individual. The best individual in a group is likely to be the optimal or approximate optimal solution of the problem.

As a new random search and optimization method to simulate biological evolution, genetic algorithm has been widely used in the field of optimization(Chen,1996; Li,2009). The parameter optimization of many empirical formulas of hydrological models is essentially based on the global optimization ability of genetic algorithm.

\subsubsection{Collins iteration method}

Iterative method is a mathematical process to solve the problem by finding approximate solutions that meet the restrictive conditions from an initial value. Iterative algorithm is also a basic method to solve problems by computer. It makes use of the characteristicof fast computing speed and suitable for repetitive operation, so that the computer can repeat a set of instructions (or steps). When the instructions (or steps) are executed, a new value of the variable will be derived from the original value of the variable. Assuming that we want to derive an approximate solution, we should determine an initial value, an iteration function and a restriction condition according to the actual situation and data firstly, until the absolute value of the initial value and the calculated approximation value is less than a certain value.That is to say, we find the exact desiredvalue.

\section{Approach used to determined the unithydrograph}

The overall calculation process is divided into two parts: (1) the parameters of the unit hydrograph are optimized using the genetic algorithm, so the initial unit hydrograph can be calculated; and (2) the final unit hydrograph is calculated using the Collins iterativemethod.

\subsection{Calculation of the initial unit hydrograph using a genetic algorithm}

(a) Parametrization of Gamma Function

For simplicity, Eqn. (10) is transformed as follows

$$
\left\{\begin{array}{l}
q_{i}={ }^{1} x-{ }^{x}(i+x) \\
q=q^{2}=0 \\
1 \quad n
\end{array}\right.
$$


Where, ${ }^{x_{1}}, x_{2}$, and ${ }^{x_{3}}$ are the constants of the unit hydrograph ${ }^{q_{i}}$, which is the argument of the problem for the genetic algorithm. $i$ is the argument of the unit hydrograph, which is a period number. $i$ is the known number, which is processed by the genetic algorithm. $\mathrm{n}$ is the time period of the unit hydrograph, which is defined according to the actual engineering problem.

The variables $x_{1}, x_{2}$, and ${ }^{x_{3}}$ are in a range of $[0,5]$. The chromosome is coded as a floating point value.

the unit hydrograph), the unit hydrograph obtained using the constant given above is asfollows.

$$
\begin{aligned}
& \text { 个. } 1.7450^{8.7014} \\
& \left\{\begin{aligned}
q_{i}=\frac{\overline{(8.7014-1)} \cdot(i+1.5042)}{} & \cdot e \\
& i=2,3, \quad, 10
\end{aligned}\right. \\
& q_{1}=q_{11}=0
\end{aligned}
$$

Using Eqn. (12), the results obtained for the unit hydrograph are [0, 570, 688, 563, 356, 187, 223 86, 35, 13, 5, 0], as shown in Fig. 2.

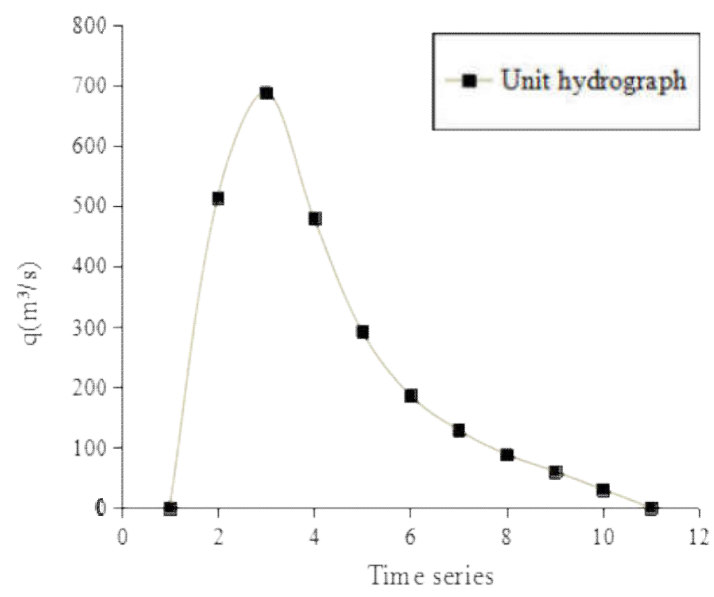

Fig. 2 Unit hydrograph $q_{i}$

(b) Determination of objective function

According to the basic principle used to derive the unit hydrograph, the objective function of the genetic algorithm can be expressed as follows:

$$
\max : \varphi\left(q_{i}\left(x, x_{3}, x_{3}\right)\right)=\frac{1}{\left(Q^{\prime}\left(q_{i}\right)-Q\right)^{2}}
$$


231 basin, and $Q$ is the measured flow in the basin outlet section.

232 Physical interpretation of the objective function Eqn. (13)

234 inverse square of the difference between $Q^{\prime}\left(q_{i}\right)$ and $Q$ is maximal. To avoid computations if

235 the objective function value is too small, the expansion coefficient $M$ is introduced. The value

236 of $M$ is determined according to the specific situation. Eqn. (13) is converted into the following 237 form.

$$
\max : \varphi\left(q_{i}\left(x_{1}, x_{2}, x_{3}\right)\right)=\frac{M}{\left(Q^{\prime}\left(q_{i}\right)-Q\right)^{2}}
$$

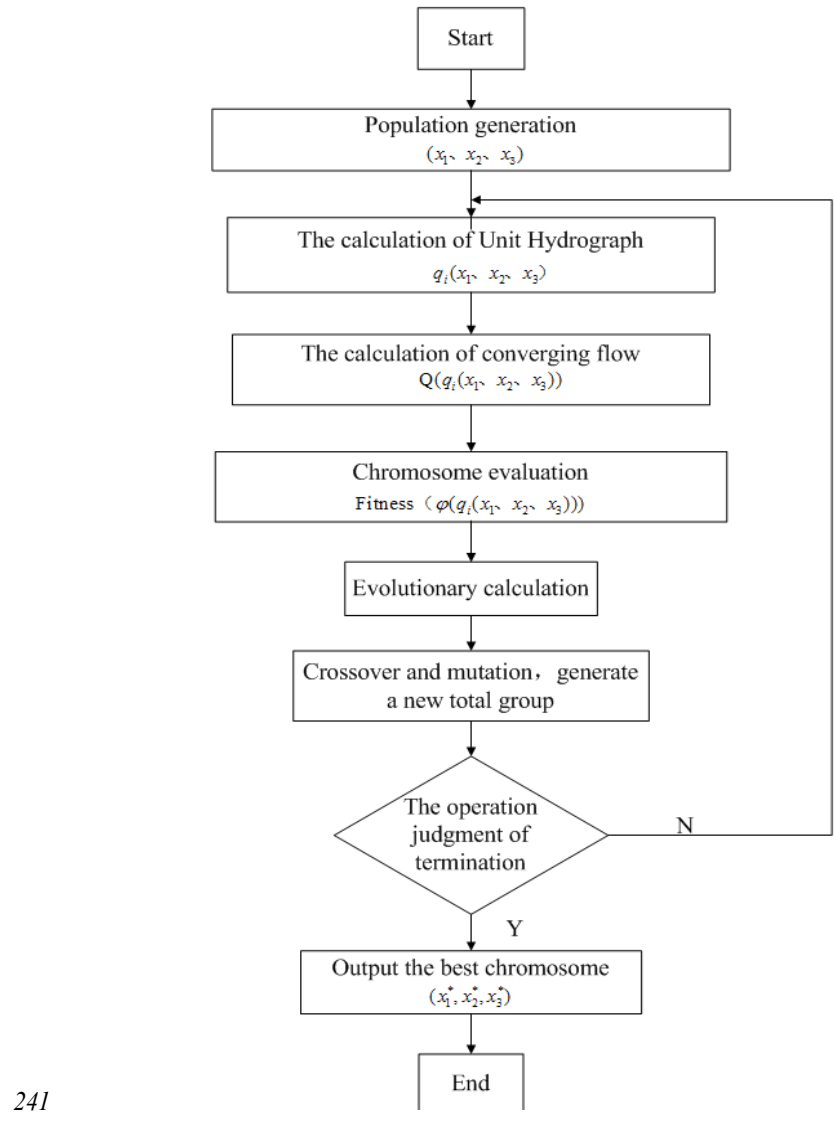




$$
\left(x^{*}, x^{*}, x^{*}\right)
$$

The optimum parameters ${ }_{1}, x_{2}$ are obtained by the above steps, and then the unit line

244 is calculated by the optimum parameters, as follows:

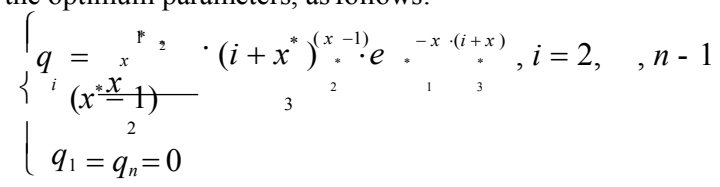

246

250 runoff $\sum Q\left(q_{i}^{*}, \overline{h_{\max }}\right)$ is calculated; Secondly, another unit hydrograph

251 $q_{i}^{\prime}=\frac{Q-\sum Q\left(q_{i}^{*}, h_{\text {max }}\right)}{h_{\max }}$ is deduced, and new $q_{i}^{\prime}$ is deduced continuously by $q_{i}^{*}$ according to $\varepsilon=\left|q_{i}^{*}-q\right| \leq$ ErrorExcepted until the error between the two

252 the restriction condition of

$$
q_{i}=q_{i}^{\prime}
$$

253 units meets the requirement, then the final unit hydrograph $q_{i}=q_{i}$.

\section{Examples}

\section{Example 1}

256

In Table 1, the data were taken from a previous study (Zhuang and Lin, 1986).

$$
\text { Table } 1 \text { The calculations of example } 1
$$

\begin{tabular}{|c|c|c|c|c|c|c|}
\hline \multirow{2}{*}{$\begin{array}{c}\text { Time } \\
\text { series(h) }\end{array}$} & \multirow[b]{2}{*}{$\mathbf{R}(\mathbf{m m})$} & \multirow{2}{*}{$\begin{array}{c}\text { The measured } \\
\text { runoff } \\
\mathbf{Q}\left(\mathbf{m}^{3} / \mathbf{s}\right)\end{array}$} & \multicolumn{2}{|c|}{$\begin{array}{c}\text { The trial and error } \\
\text { method }\end{array}$} & \multicolumn{2}{|c|}{ GACIM } \\
\hline & & & $Q^{\prime}\left(\mathbf{m}^{3} / \mathbf{s}\right)$ & $q^{\prime}\left(\mathrm{m}^{3} / \mathrm{s}\right)$ & $Q^{\prime \prime}\left(\mathbf{m}^{3} / \mathbf{s}\right)$ & $q^{\prime \prime}\left(\mathbf{m}^{3} / \mathbf{s}\right)$ \\
\hline (1) & (2) & (3) & (4) & (5) & (6) & (7) \\
\hline 0 & & 0 & 0 & 0 & 0 & 0 \\
\hline 6 & 3.8 & 0 & 0 & 0 & 0 & 0 \\
\hline 12 & 3.9 & 50 & 190 & 500 & 195 & 514 \\
\hline 18 & 0 & 252 & 455 & 685 & 461 & 687 \\
\hline 24 & 27.3 & 662 & 446 & 470 & 450 & 480 \\
\hline 30 & 2.9 & 1700 & 1650 & 280 & 1700 & 292 \\
\hline
\end{tabular}

R: unit hydrograph; Q: measured discharge; $Q^{\prime}, q^{\prime}$ : discharge and unit hydrograph of trial and error method;

259 discharge and unit hygrograph of GACIM 
https://doi.org/10.5194/npg-2020-1

Preprint. Discussion started: 1 April 2020

(c) Author(s) 2020. CC BY 4.0 License.

\begin{tabular}{lccccc}
36 & 2210 & 2200 & 195 & 2210 & 186 \\
42 & 1610 & 125 & 1630 & 129 \\
48 & 1630 & 981 & 85 & 1020 & 88 \\
54 & 1020 & 669 & 60 & 650 & 60 \\
60 & 650 & 433 & 35 & 440 & 30 \\
66 & 440 & 288 & 15 & 290 & 0 \\
72 & 290 & 195 & 0 & 190 & \\
78 & 190 & 113 & & 100 & \\
84 & 100 & 51 & & 9 & \\
90 & 40 & 4 & & 0 & \\
\hline
\end{tabular}

260

261 The unit hydrographs determined using the two methods are shown in columns (5) and (7) in

262 Table 1. A comparison of the unit hydrographs is shown in Figure 4. The flow processes

263 calculated using the unit hydrographs are shown in columns (4) and (6) in Table 1. A comparison

264 between the calculated flow process and the measured flow is shown in Figure5.

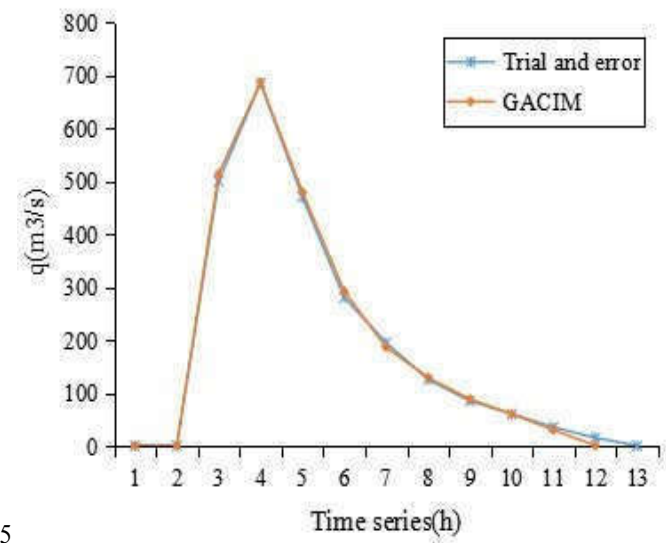

266

Fig. 4 Example1: Unit hydrographs ascertained by two methods 


\begin{tabular}{|c|c|c|}
\hline Project Method & GACIM & The trial and error \\
\hline The error of flood peak $\left(\mathrm{m}^{3} / \mathrm{s}\right)$ & 0 & 10 \\
\hline The maximum error of discharge( $\left.\mathrm{m}^{3} / \mathrm{s}\right)$ & 212 & 216 \\
\hline The average absolute error of discharge $\left(\mathrm{m}^{3} / \mathrm{s}\right)$ & 37.31 & -51 \\
\hline The total error of flood peak discharge( $\left.\mathrm{m}^{3} / \mathrm{s} . \mathrm{h}\right)$ & -111 & -0.55 \\
\hline The relative error of flood peak discharge $(\%)$ & -1.20 & \\
\hline
\end{tabular}

278

279

The actual hydrological data in Table 1 show that the period number for the runoff was 5 and the period number for the flow process in the outlet section was 15 . According to the theory of the unit hydrograph, the period number for the unit hydrograph should be $15-5+1=11$. Using the GACIM (genetic algorithm and the Collins iterative method), the period number for the unit hydrograph was 11 . Using the trial and error method, the period number for the unit hydrograph was 12. To further consider the performance of the two methods, we compared the flow process in the outlet section and the results obtained using the two unit hydrographs (measured value and calculated value). A statistical analysis of the results is shown in Table2.

\section{Example 2}

In Table 3, the data were taken from a previous study (Li and Zheng, 1982).

Table 3 The calculations of example 2

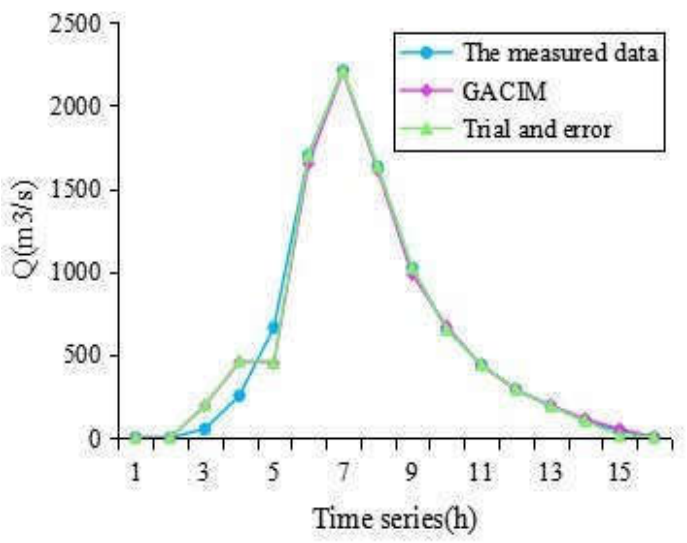

Fig. 5 Example1: The flow process of outletsection

\begin{tabular}{|c|c|c|c|c|}
\hline $\begin{array}{c}\text { Time } \\
\text { series(h) }\end{array}$ & $\mathbf{R}(\mathbf{m m})$ & $\begin{array}{l}\text { The measured } \\
\text { runoff } Q\left(\mathrm{~m}^{3} / \mathrm{s}\right)\end{array}$ & $\begin{array}{c}\text { The trial and error } \\
\text { method }\end{array}$ & GACIM \\
\hline
\end{tabular}




\section{$Q^{\prime}\left(\mathbf{m}^{3 / \mathbf{s}}\right) \quad q^{\prime}\left(\mathbf{m}^{3 / \mathbf{s}}\right) \quad Q^{\prime \prime}\left(\mathbf{m}^{3 / \mathbf{s}}\right) \quad q^{\prime \prime}\left(\mathbf{m}^{3 / \mathbf{s}}\right)$}

\begin{tabular}{ccccccc}
\hline 1 & $(2)$ & $(3)$ & $(4)$ & $(5)$ & 6 & (6) \\
\hline 0 & & 0 & 0 & 0 & 0 & 0 \\
6 & 153 & 97 & 96 & 63 & 97 & 49
\end{tabular}

97

214

12

18

5.8

24

24

30

36

42

48

54

60

66

72

The unit hydrographs determined using the two methods are shown in columns (5) and (7) in

Table 3. A comparison of the unit hydrograph is shown in Figure 6. The flow processes calculated

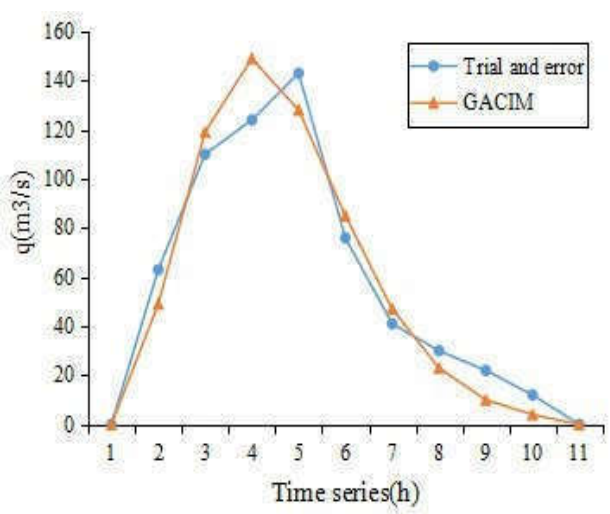

Fig. 6 Example2: Unit hydrographs ascertained by two methods 


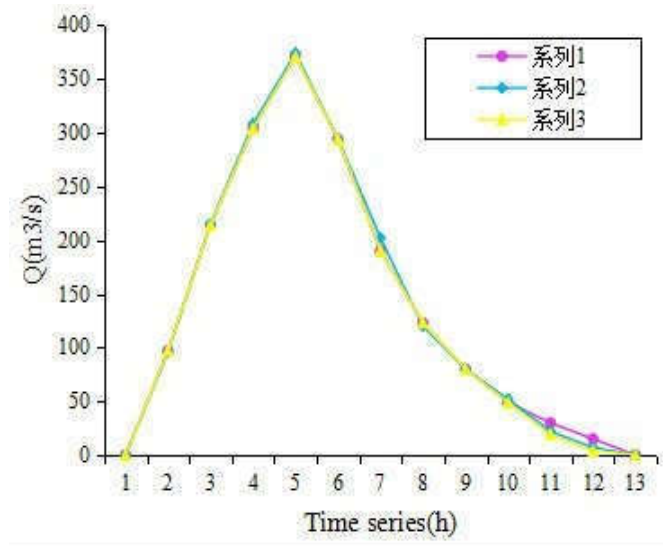

Fig. 7 Example2: The flow process of outletsection

Figure 4 shows that GACIM was significantly better than the trial and error method in terms of the shape of the curve. We also compared the flow process in the outlet section and the data obtained using the two unit hydrographs. A statistical analysis of the results is shown in Table4.

Table 4 The error statistics of example2

\begin{tabular}{|c|c|c|}
\hline Project Method & GACIM & The trial and error \\
\hline The error of flood peak(m3/s) & 0 & -3 \\
\hline The maximum error of discharge(m3/s) & 11 & -12 \\
\hline The average absolute error of discharge(m3/s) & 1.69 & -0.23 \\
\hline The total error of flood peak discharge(m3/s.h) & 22 & -3 \\
\hline The relative error of flood peak discharge (\%) & 1.25 & -0.17 \\
\hline
\end{tabular}

From Table 2 and Table 4, the calculation accuracy of BGACM is obviously better than that of trial-and-error method in most projects. Although the total error of flood volume is larger than that of trial-and-error method, the relative error of flood volume is only $1.2 \%$ and $1.25 \%$, so it does not affect the application of actual projects.

Figure 6 and 7 show that the flow processes of the two unit hydrographs weresimilar. However, a comparison of the shapes of the unit hydrograph showed that the continuity and smoothness of GACIM were better than the trial and error method. The GACIM method conformed better with the features of a time-invariantsystem.

It can be seen that BGACM method is better at simulating river basin confluence process, which depends on the physical mechanism of the algorithm, while trial-and-error method pays more attention to the balance of total flood volume. This is the respectivecharacteristics and advantages of the two algorithms exactly. 


\section{Discussion}

The present study used a combination of a genetic algorithm and the Collins iterative method (GACIM) for determining a unit hydrograph. The method and implementation steps were described, while examples and analyses were used to demonstrate the scientificity, reliability and practicability of this method. The outcomes of this study are discussedbelow.

(a) Difference between GACIM and other methods

In principle, GACIM is based on composition theory and it describes the physical mechanism and process of flood confluence using mathematical equations. Using the basic concept of the unit hydrograph and a genetic algorithm as a mathematical tool, this method can be used to simulate the flood confluence process.

Therefore, the simulation of the convergence process is more accurate with GACIM.

Other methods for calculating unit hydrographs include the analysis method, least squares method, and the trial and error method. These methods are more focused on the unit hydrograph as an outlet flow process and they fit the measured flow precisely, but they ignore the composition and structure of the unit hydrograph itself. Example 2 shows that GACIM performed better at simulating the basin confluence process, whereas other methods paid more attention to the balance of the total flood volume.

(b) Genetic operator design issues

A genetic algorithm is a very useful optimization tool. Its biggest advantage is that it has wide adaptability and unlimited problem space, so it can handle many different constraints. This strategy uses a penalty factor. This is because the genetic algorithm method delivers exhaustive engineering accuracy if the population is sufficiently large.

There are two types of genetic algorithm, i.e., the standard genetic algorithm (crossover and mutation) and evolutionary computing (selection). A genetic algorithm simulates the recombination of genes to create new offspring in each generation, whereas evolutionary computation is a population process that updates each generation.

In this study, a genetic algorithm was used to optimize the parameters of the Gamma function and the unit hydrograph was calculated according to the law of basin confluence. Thus, the parameters were generated by a genetic algorithm. Therefore, the design of the genetic operators is related directly to whether reasonable generation parameters could be obtained.

A genetic algorithm has two components: crossover and mutation. Crossover is the main genetic operation that generates new individuals, but it also maintains the relative stability of the population at the same time. However, the variation is a basic calculation and the main effect is to produce a new gene from the population, which provides new information for thepopulation.

In general, the initial population of the genetic algorithm is generated in the value space. Crossover and mutation are performed in the value space. In the present study, the value of the Gamma function was in a certain range. Initially, we could not define a reasonable space. If the value space is too large, a bigger population must be used to meet the needs of the individual 
distribution density. However, this greatly reduces the computational speed. If the value space is too small, it might not meet the parameter combination required for the engineering precision. To solve this problem, we observed the following principles during the design of the geneticoperator.

(i) The crossover operator was determined where a new individual was generated at random in the space $[0, a](a>0)$.

(ii) The random expansion value space of the mutation operator was [0 a $]$ and its amplitude was random. For the floating point coding mutation operator, the following program was implemented in MATLAB:

1) numMut=round(size(parent,1)*Ops/2); to calculate the number of variations

2) numPop=size(parent,1); to calculate the size of the population

3) numPara $=$ size(parent,2); to calculate the number of parameters

4) for $\mathrm{j}=1$ :numPara

5) for $i=1$ :numMut

6) $\mathrm{a}=$ round(rand*(numPop-2)+2); select a male parent

7) parent $(\mathrm{a}$, numPara $)=\operatorname{parent}(\mathrm{a}$, numPara $) *(1+\mathrm{rand} /$ gen $)$; generate a new generation

8) end

9) end.

The parameters of the offspring chromosome were calculated during step 7) of this program, where the variation in the amplitude was related to the number of evolutionary passages. The variation in the amplitude declined gradually with increasing passagenumbers.

Figure 8 illustrates the crossover operator and mutation operator with the passage of evolutionary time.

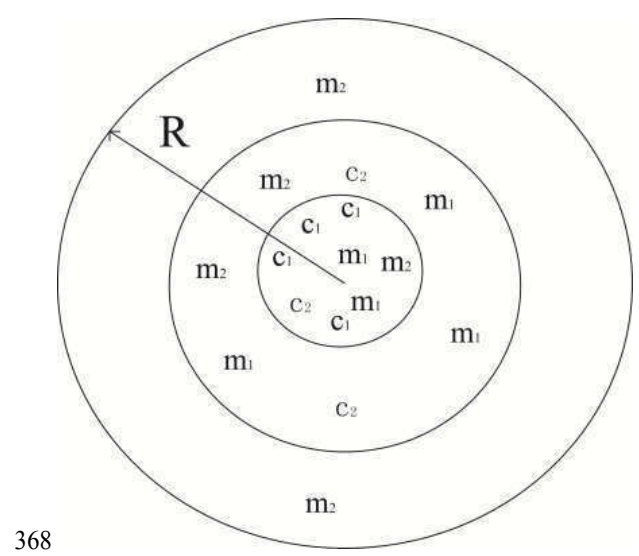

Fig. 8 The sketch map of the continuation of parameters valuespace

In the first generation, the filial generation caused by the crossover operator was still in the initial parameter space, which corresponds to the inner loop in Figure 8. However, the filial 
ring. In the second generation, the filial generation of the crossover operator was extended to the second ring while the filial generation of the mutation operator was extended to the third ring. The expanding amplitude of the adjacent ring decreased with the passage of evolutionary time. This method was repeated until the predetermined evolutionary algebra was completed.

In Examples 1 and 2, the initial values of the parameters were [l $\left.\begin{array}{ll}0 & 5\end{array}\right]$. The two sets of optimized parameters were as follows.

Example 1: [1.7450, 8.7014, 1.5042]

Example 2: [1.6052, 7.9209, 0.30007]

These two examples demonstrate the design rationality and the validity of the genetic operator.

(c) Research methods for hydrological analysis and calculation

The factors that affect hydrological phenomena are very complex. There is still no accurate understanding of the causal relationships among hydrologic phenomena. It is considered that hydrological phenomena involve certainty and randomness, which form the basis of hydrological research. Therefore, causal analysis and probabilistic statistics are the main methods used for hydrological analysis and calculation. In practical applications, causal analysis is confined mostly to qualitative analysis. Quantitative problems demand empirical statistical relationships based on actual observational data.

Based on the theory of composition, the distribution function in statistical physics has been extended to hydrology as a non-physics field. Thus, hydrological systems can be viewed as a generalized collection. The regularities of hydrological phenomena have been simulatedusing distribution functions. Distribution functions and functional relationships have been determined using observation data, which generally means that objective laws are formalized.

The present study was a preliminary attempt to investigate the quantitativerelationships among hydrological phenomena based on the theory of composition and its distribution function. The author believes that this theory could be a new approach to exploring hydrologicalrules.

Author Contributions: Hongyan Li conceived and designed the paper; Cidan Yangzong analyzed the data; Hongyan Li and Cidan Yangzong wrote the paper.wrote the paper.

Foundation item: The author hereby would like to express deep gratitude to the key special project of "Efficient 399 Development and Utilization of Water Resources" (2017YFC0406005), China-ROK cooperation project (51711540299), 400 Natural Science Foundation of Jilin Province(20180101078JC) and other projects which have given support to the 401 research of this paper.

Acknowledgments: The authors hereby would like to express deep gratitude to the key special project of "Efficient Development and Utilization of Water Resources" (2017YFC0406005), China-ROK cooperation project (51711540299), Natural Science Foundation of Jilin Province (20180101078JC) and other projects which have given support to the research of thispaper.

Data Availability Statement: The data sources and code used are provided in the article 


\author{
411 References \\ 1. Bhunya P K, Mishra S K, Berndtsson R. Simplified two-parameter gamma distribution \\ for derivation of synthetic unit hydrograph[J]. Journal of Hydrologic Engineering, 2003, \\ 8(4):226-230. \\ 2. Bhunya P K, Berndtsson R, Ojha C S P, et al. Suitability of Gamma, Chi-square,Weibull, \\ and Beta distributions as synthetic unit hydrographs[J]. Journal of Hydrology, 2007,334(1): \\ 28-38. \\ 3. Bobbitt P. Constitutional fate: Theory of the Composition[M]. New York: Oxford \\ University Press, 1982. \\ 4. Collins W T. Runoff distribution graphs from precipitation occurring in more than one \\ time unit[J]. Civ. Eng.(NY), 1939, 9(9): 559-561. \\ 5. Cui H, Singh V P. On the cumulative distribution function for entropy-based hydrologic \\ modeling[J]. Transactions of the ASABE, 2012, 55(2):429-438. \\ 6. Dooge J. Linear theory of hydrologic systems[M]. Agricultural Research Service, US \\ Department of Agriculture, 1973. \\ 7. Johnstone D, Cross W P. Elements of applied hydrology[M]. Ronald Press Company, \\ 1949. \\ 8. Li, H.L., Zheng, Z.Q.(1982). Hydrological forecast and calculation[J]. Beijing:China. \\ Water Resources and Electric Power Press,(in Chinese) \\ 9. Raghunath H M. Hydrology: principles, analysis and design[M]. New AgeInternational, \\ 2006. \\ 10. Ramirez J A. Prediction and modeling of flood hydrology and hydraulics[J]. Inland \\ Flood hazards: Human, riparian and aquatic communities, 2000: 293-333. \\ 11. Sherman L R K. Streamflow from rainfall by the unit-graph method[J]. Eng. News \\ Record,1932, 108: 501-505. \\ 12. Singh S K. Clark's and Espey's unit hydrographs vs the gamma unithydrograph/Les \\ hydrogrammes unitaires de Clark et de Espey vs l'hydrogramme unitaire de formeloi \\ gamma[J]. Hydrological sciences journal, 2005, 50(6). \\ 13. Singh S K. Time Base as an Invertible Function of the Parameters of GammaUnit \\ Hydrograph[J]. Journal of Irrigation and Drainage Engineering, 2009, 135(6): 802-805. \\ 14. Hanson T.L and Johnson H.P. Unit Hydrograph Methods Compared[J] Transactions of \\ The ASAE, 1964.448-451. \\ 15. Viessman Jr W. The dynamics of water policy[J]. Water Management, 1989.41-47. \\ 16.Ye, S.J., Xia, J. Century's retrospect and looking into the future of hydrological \\ science[J] Advence in Water Science,2002,13(1): 93-104 (inChinese). \\ 17. Yuan, J., Cheng, Y.B., Zhao, Y.F. The parabolic unit hydrograph model offlood \\ forecasting of river course in Gezhou Dam[J]. Yangtze River. 1991,6-7.(inChinese) \\ 18. Zhai, G.J., Li, Y.Q. Math-model and Its Parameter Recognition of the Finite-period \\ Unit Line[J]. Journal of Engineering and Techincal Higher Specialty Institutions, \\ 2004,1:001. (in Chinese). \\ 19. Zhang, W.X. The theory of composition [M]. Hefei: China University of Science and \\ Technology of China press, 2003 (in Chinese). \\ 20.Davis, L. Handbook of genetic algorithms[M]. New York :Van Nostrand Reinhold,1991. \\ 21. Michalewicz \& Zbigniew. Genetic algorithms + data structures = evolutionprogram. \\ Berlin:Springer-Verlag(1996). \\ 22. Yuan ximin,Li hongyan,Liu shukun,etc. Application of Neural Network and Genetic \\ Algorithms in Water Science[M]. Beijing:China Water Resources andHydropower
}


https://doi.org/10.5194/npg-2020-1

Preprint. Discussion started: 1 April 2020

(C) Author(s) 2020. CC BY 4.0 License.

458 Publishing House, 2002(in Chinese).

23. Chen guoliang, Wang xufa, Zhuang zhenquan, etc. Genetic Algorithms and Their Applications[M]. Beijing: People's Posts and Telecommunications Publishing House,

$461 \quad$ 1996(in Chinese).

24. LI hongyan, Liu xiaowei ,Li shiming . Application of the optimum empirical formula By GAS to the calculation of thesediment concentration process in the Lower Yellow River[J].Journal of Sediment Research, 2009, 30(3):30-36. 